After four perfusions (weeks $0,2,6,10$ ) clinical improvement (healing of skin necrosis, reduced dyspepsia and dyspnoe, increased general feeling, reduced VAS from 80 to 20, increased opening of the mouth, increased movement of the hands and fingers) permitted a slow reduction of the daily steroid dose.

Objectives ./.

Methods ./.

Results ./.

Conclusion This is a case with positive therapeutic onset of infliximab (remicade) in a case with systemic sclerosis.

\section{FRI0184 ANGIOTENSIN-CONVERTING ENZYME AND ANTI- ANGIOTENSIN-CONVERTING ENZYME ANTIBODIES IN SYSTEMIC SCLEROSIS}

RT Alekperov, ML Stanislav, MA Myagkova, TV Abramenko, OA Kost, II Nikolskaya. Microcirculation, Institute of Rheumatology of RAMS, Moscow, Russia

\subsection{6/annrheumdis-2001.265}

\section{Background}

Objectives To investigate the levels of angiotensin-converting enzyme (ACE) and anti-angiotensin-converting enzyme antibodies (ACEab) in patients with systemic sclerosis (SSc).

Methods Thirty five patients with systemic sclerosis, age 50,0 \pm 11,7 (mean $\pm \mathrm{SD}$ ), range $27-71$ years, were included in the study. Duration of disease ranged from 1 to 20 years, means 7,0 $\pm 5,2$. Thirteen pts had diffuse systemic sclerosis ( $\mathrm{dSS} c$ ), twenty two had limited systemic sclerosis (ISSc). Ten healthy subjects were selected as controls. Spectrofluorimetry for detection of ACE and ELISA for ACEab were used.

Results We revealed the two-fold lower levels of ACE in SSc pts compared with controls $(0,022 \pm 0,021$ vs $0,044 \pm 0,028$; ð= 0,012). There were no differences in ACE values observed between the dSSc and 1SSc groups $(0,019 \pm 0,017$ and 0,024 \pm 0,024 respectively). The lowest levels of ACE were found in SSc pts treated with inhibitors of ACE $(0,013 \pm 0,010)$, but was not statistically different from those of untreated SSc pts. In SSc pts, a positive correlation was detected between the levels of ACE and erythrocyte sedimentation rate $(\mathrm{r}=0,505 ; \mathrm{p}=0,019)$, fibrinogen $(\mathrm{r}=0,578 ; \mathrm{p}=0,015)$, titers of antinuclear antibodies $(\mathrm{r}=0,529 ; \mathrm{p}=0,029)$.

The levels of ACEab in SSc pts were similar to those of the control group $(0,180 \pm 0,190$ and 0,208 $\pm 0,057$, respectively). However, the ACEab levels in pts with dSSc were significantly higher than those in $1 S S c$ pts $(0,29 \pm 0,24$ vs $0,14 \pm 0,16 ; \mathrm{p}=$ 0,041). Comparable to $1 S S c$ group impaired results were also seen in pts treated with ACE inhibitors $(0,1642 \pm 0,1093)$. The ACEab levels positively correlated with ANA titers only in dSSc pts $(r=0,835 ; p=0,019)$. There was no relation between the ACE and ACEab levels neither in SSc pts nor in controls.

Conclusion ACEab can be involved in pathogenesis of SSc and further investigation is needed.

\section{FRI0185 AUTOLOGOUS STEM CELL TRANSPLANTATION INTERNATIONAL SCLERODERMA TRIAL}

${ }^{1} \mathrm{JM}$ Van Laar, ${ }^{2} \mathrm{D}$ Farge, ${ }^{3} \mathrm{~A}$ Tyndall. On Behalf of the EBMT/EULAR Scleroderma Study Group; 'Department of Rheumatology, Leiden University Medical Center, Leiden, The Netherlands; ${ }^{2}$ Department of Internal Medicine, Hopital Saint-Louis, Paris, France; ${ }^{3}$ Department of Rheumatology, Felix-Platter Spital, Basel, Switzerland
Background Autologous hemopoietic stem cell transplantation (HSCT) is emerging as a potential therapy for severe rheumatic autoimmune diseases, including systemic sclerosis. Phase I/II studies involving 65 patients with progressive SSC and organ involvement proved the feasibility of HSCT and demonstrated significant longterm effects on skin score, but with considerable toxicity including a treatment-related mortality of $12 \%$. These results must be viewed against current data on morbidity and mortality (5-yr survival 50\%) for this patient category. At the recent EBMT/EULAR consensus meeting, the concept for a randomised, controlled trial was approved to investigate whether the longterm benefits of HSCT outweigh the risks. As a result the ? ASTIS Trial? (Autologous Stem cell Transplantation International Scleroderma Trial) has now been launched.

Objectives To compare efficacy and safety of HSCT versus monthly intravenous pulse-therapy cyclophosphamide in patients with diffuse systemic sclerosis and heart, lung or kidney involvement at risk for premature mortality. The goal of both treatments is to prolong survival by arresting or retarding the disease process. It is postulated that HSCT has superior efficacy, which needs to be balanced against potentially higher toxicity.

Methods The ASTIS Trial is an international, multicenter, prospective, controlled, randomised, phase III study in selected patients with early diffuse SSC and major organ involvement. The ASTIS Trial is conducted under the auspices of EBMT/ EULAR (Basel, Switzerland). The investigational treatment arm (HSCT) comprises the following consecutive steps: mobilisation of hematopoietic stem cells with i.v. cyclophosphamide $(2 \times 2$ $\left.\mathrm{gr} / \mathrm{m}^{2}\right)$ and filgrastim $(10 \mathrm{ug} / \mathrm{kg} /$ day $)$, leukapheresis and selection of $\mathrm{CD}_{34}{ }^{+}$stem cells, conditioning with i.v. cyclophosphamide $(200 \mathrm{mg} / \mathrm{kg}$ ) and rbATG $(7.5 \mathrm{mg} / \mathrm{kg})$, followed by reinfusion of autologous stem cells. The control treatment arm consists of 12 monthly i.v. pulses cyclophosphamide $\left(750 \mathrm{mg} / \mathrm{m}^{2}\right)$.

The primary endpoint is event-free survival during the study period of 2 years, events defined as the development of persistent major organ failure (heart, lung, kidney) and death. Secondary endpoints include treatment-related mortality, toxicity, skin score, SHAQ, and quality-of-life. Side studies include evaluation of immune reconstitution in blood and fibrotic activity in skin. It is intended to enrol 200 patients in 3 years.

Results

Conclusion The ASTIS Trial is the first randomised study to evaluate the potential clinical benefits of HSCT versus conventional therapy in severe rheumatic autoimmune disease. The study will address whether a short period of intense immune suppression differs from chronically given, more moderate immune suppression, bot qualitatively and quantitatively. Given the low incidence of severe forms of systemic sclerosis, and the efforts needed to conduct the study, international collaboration is mandatory.

\section{FRI0186 CIRCADIAN PROFILE OF SERUM MELATONIN IN PATIENTS WITH SYSTEMIC SCLEROSIS}

${ }^{1}$ AT Kotulska, ${ }^{2}$ L Brzezinska-Wcislo, ${ }^{1} E J$ Kucharz. 'Internal Medicine and Rheumatology; ${ }^{2}$ Dermatology, Medical University of Silesia, Katowice, Poland

\subsection{6/annrheumdis-2001.267}

Background Melatonin (MT) is secreted by the pineal gland and a circadian rhythm of MT secretion is related to light-darkness exposure. MT has been found to influence immune and endocrine functions and is a free radical scavanger. Immune 
abnormalities and disturbed endocrine secretion have been reported in patients with systemic sclerosis (SSc). Additionally, some of the patients have sleep disturbances or depression, a factors known to effect the MT level. All these findings suggest possible role of MT in pathogenesis of some phenomena seen in SSc patients.

Objectives The aim of the study was to evaluate the circadian profile of serum MT levels in patients with SSc.

Methods Six women with definite SSc, aged $32.4+1.8$ yrs (symptoms duration 4-7 yrs) and six age-matched healthy women were investigated. Blood samples were drawn at 8.00, $12.00,16.00,20.00,22.00,24.00,02.00,04.00$. 06.00. Light exposure of the subjects was from 07.30 to 21.00. MT was determined with ELISA method.

Results A significant circadian rhythm of serum MT was detected in all investigated subjects. Serum MT levels were lower in the SSc patients than in the controls, MESOR $=21.4 \mathrm{pg} / \mathrm{ml}$ and $47.3 \mathrm{pg} / \mathrm{ml}$, in the patients and controls, respectively. In the SSc patients, the night increase in MT was also relatively lower when compared to the daily levels. Maximal values were found between $02.00-04.00$ both in the patients and controls.

Conclusion The general nature of the circadian serum MT rhythm is maintained in the SSc patients although secretion of MT is depressed. The mechanism of impaired MT secretion remains unknown. It may be speculated that a low MT level is involved in development of immune abnormalities, neuroendocrine disfunctions and/or impaired free radical elimination. All these phenomena may contribute to development of fibrosis.

\section{FRI0187 THE CLINICAL PROFILE OF JUVENILE SYSTEMIC SCLEROSIS (JSSC)}

MN Starovoytova, NG Guseva. Depatment of Microcirculation and Inflammation, Institute of Rheumatology, RAMS, Moscow, Russia

\subsection{6/annrheumdis-2001.268}

Background JSSc represents the unique form of SSc characterised by variable $\mathrm{f}$ clinical picture. JSSc has specific features maintained during adult period of life.

Objectives The aim of the study was to determine the clinical profile of JSSc.

Methods Sixty pts with JSSc aged from 14 to 54 years (mean $25.1+7.2 \mathrm{yrs}$ ) with the onset of the disease at the age of 1 to 16 years (mean $11.4+3.8$ yrs) were analysed. Duration of disease was $13.1+7.1$, range $1-39$ yrs.

Results We revealed three clinical variants of JSSc. The first variant (55\% of pts) is characterised by the combination of localised scleroderma (morfhea, hemiform-typed) and systemic manifestation such as Raynaud's phenomenon (78\%), arthritis (41\%), lungs involvement (66\%), heart disease $(58 \%)$, gastrointestinal tract involvement $(60 \%)$. The visceral signs were moderate and founded by careful clinical instrumental investigation. In the other variant $(38 \%$ pts) the clinical manifestation of JSSc were similar to adult $1 S S c$ with the prevalence of vascular disorders and digital ischaemic changes, limited skin lesion, visceral organ involvement. Musculoskeletal manifestations (arthralgia, arthritis, flexon contractures) were also seen in this group. The pts hadeslow progressive form of disease. The third variant (17\% of pts) may be regarded as overlap syndrome SSc with polymiositis or/ and rheumatoid arthritis. The state in these pts mainly depended on the prevailing disease process.
Conclusion The definition of the clinical variants of JSSc may provide the correct diagnosis and adequate treatment.

\section{FRI0188 DIASTOLIC DYSFUNCTION IN PATIENTS WITH SCLERODERMA: A TISSUE DOPPLER STUDY}

A Dinc, M Uzun, S Pay, I Simsek, F Kilicarslan, H Erdem. Department of Internal Medicine, Division of Rheumatology, and Department of Cardiology, Gülhane School of Medicine, Ankara, Turkey

\subsection{6/annrheumdis-2001.269}

Background Systemic sclerosis may involve myocardium and brings about myocardial fibrosis.

Objectives In our study, left ventricular diastolic functions were evaluated with the tissue Doppler echocardiography, a newer and more sensitive method than others.

Methods Standard and tissue Doppler echocardiography were applied to 11 patients with systemic sclerosis. Systolic and valve functions were evaluated with standard echocardiography and then the signals obtained from the lateral section of mitral annulus were analysed with pulse wave tissue Doppler echocardiography. Left ventricular diastolic functions were assessed by calculating early wave (E), atrial wave (A) and E/A ratio of these signals.

Results The impairment of left ventricular diastolic functions was determined in 4 patients. This finding was not related to the duration of disease and the spread of involvement. All patients have normal ejection fractions.

Conclusion Left ventricular diastolic dysfunction is a newly defined special feature for cardiac involvement of systemic sclerosis. This finding may be due to myocardial ischemia, fibrosis, or both of them. However, larger studies are necessary to evaluate the prognostic importance of this finding.

\section{FRI0189 PROGNOSTIC SIGNIFICANCE OF LIMITED AND DIFUSSE FORM OF SKIN CHANGES IN SYSTEMIC SCLEROSIS}

NS Damjanov, G Dobrosavljevic, S Pavlov, G Radunovic, I Vukovic, N Todorovic, M Milenkovic, P Ostojic. Clinical II, Institute of Rheumatology, Belgrade, Yugoslavia

10.1136/annrheumdis-2001.270

\section{Background}

Objectives To assess the difference in frequency of pulmonary and renal involvement, as well as lungs insufficiency and renal failure between patients (pts) with limited cutaneous Systemic Sclerosis (lcSSc) and difusse cutaneous Systemic Sclerosis (dcSSc). Methods Group of 138 pts with Systemic Sclerosis (SSc), 127 females and 11 males, age 28-75 years (median 53), were followed for 3-26 (median 13) years period. Extent of skin involvement was recorded, and pts were examined for radiographic signs of pulmonary fibrosis, carbon monoxide transfer (TCO) factor (done in 28 pts), and renal involvement and failure (urine sediment, creatinin clearance, arterial tension). Difference of visceral involvement, pulmonary insufficiency and renal failure between pts with $\mathrm{lcSSc}$ and $\mathrm{dcSSc}$ was correlated.

Results Among 138 pts, 105(76\%) had lcSSc, and 33(24\%) dcSSc. Pulmonary lession were found in $40 / 105$ pts with lcSSc, and in $25 / 33$ pts with dcSSc ( $\mathrm{p}=0,004-\chi 2$ test). Renal lession was detected in $11 / 105$ pts with lcSSc, and in $8 / 33$ pts with $\mathrm{dcSSc}(\mathrm{p}=0,04-\chi 2$ test). Signs of lung insufficiency developed in $30 / 105$ pts with $\mathrm{lcSSc}$, and in $18 / 33$ pts with dcSSc ( $\mathrm{p}=$ $0,04-\chi 2$ test). Lower creatinin clearance and high arterial blood 